\title{
Fibre-optic SPR sensor with a FBG interrogation scheme for readout enhancement
}

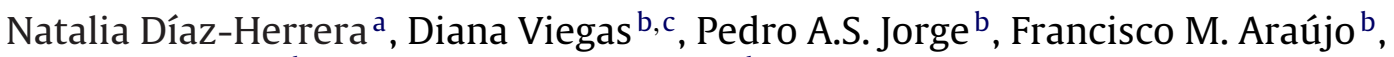 \\ José-Luis Santos ${ }^{\mathrm{b}, \mathrm{c}}$, María-Cruz Navarrete ${ }^{\mathrm{d}}$, Agustín González-Cano ${ }^{\mathrm{a}, *}$ \\ a Department of Optics, Escuela Universitaria de Óptica, Universidad Complutense, Arcos de Jalón 118, 28037 Madrid, Spain \\ b INESC Porto, Rua do Campo Alegre 687, 4169-007 Porto, Portugal \\ ${ }^{c}$ Department of Physics, FCUP, Rua do Campo Alegre 687, 4169-007 Porto, Portugal \\ ${ }^{\mathrm{d}}$ Department of Optics, Facultad de Ciencias Físicas, Universidad Complutense, Ciudad Universitaria, 28040 Madrid, Spain
}

\section{A R T I C L E I N F O}

\section{Article history:}

Received 7 July 2009

Received in revised form 13 October 2009

Accepted 23 October 2009

Available online 30 October 2009

\section{Keywords:}

Fibre-optics sensors

Fibre Bragg gratings

Surface plasmon resonance

\begin{abstract}
A B S T R A C T
In this work a new configuration of a refractometric sensor for aqueous solutions based on the combination of surface plasmon resonance (SPR) with fibre Bragg gratings (FBG) is presented. Two FBGs are selected having reflection maxima in each side of the plasmon resonance peak. These FBGs enable a different processing scheme for the information provided by the SPR transducer. This improved interrogation method increases the sensitivity and resolution of the sensor compared with those obtained with the usual method of tracking the spectral transmittance minimum and makes the system performance independent of optical source power fluctuations. The experimental results obtained with a doublelayer uniform-waist tapered fibre show the feasibility of this approach and its applicability in SPR-based biosensors that must face very exigent measuring conditions.
\end{abstract}

(c) 2009 Elsevier B.V. All rights reserved.

\section{Introduction}

Surface plasmon resonance (SPR) has become a standard technique for a huge variety of chemical sensors or biosensors, mainly in the so-called Kretchmann configuration, although tapered optical fibres (TOFs) have been accepted as well as a useful alternative to those configurations in these fields [1-3]. Plasmon excitation strongly depends on the refractive index of the surrounding medium, and, in that sense, all these sensors can be considered refractometers. In the SPR phenomenon it is also very critical the polarization of the incident light because only TM-polarized incident light will excite plasmons. The plasmon excitation produces a minimum in the spectral transmittance for a certain wavelength and refractive index values.

At the same time, the basis and properties of fibre Bragg gratings (FBGs) are well known after an intense research work in the field in the last decade $[4,5]$. It has been proved their great versatility and usefulness when used as sensors in many different areas, from structural monitoring to chemical sensing passing through fibre-optic communications [6,7]. In a FBG the periodic modulation of the fibre core refractive index makes this structure a narrow wavelength filter. Only wavelengths within a short width window

\footnotetext{
* Corresponding author. Tel.: +34 913946903; fax: +34 913946885.

E-mail address: agus@fis.ucm.es (A. González-Cano).
}

centred at the Bragg wavelength are reflected. This is the key feature to implement the interrogation scheme proposed here.

Although both SPR and FBG are concepts of great interest in the field of fibre-optic sensors, there are not too many references in which their potentialities are usefully combined. FBGs have been used to excite surface plasmons, or for temperature compensation [8-11]. But, on the other hand, FBGs have not been very commonly used as basis of advanced interrogation techniques, despite the many possibilities they offer. We propose in this work a new concept of a combined SPR/FBG sensing system in which a readily deployable interrogation technique permits to reduce or overcome some of the most usual problems that one finds in real applications of plasmon-based sensors, namely, complexity in the readout of the SPR sensor and its dependence on the power fluctuations along the optical system, as well as on the light polarization fluctuations, factors that very often limit the achievable measurand resolutions.

\section{Material and methods}

\subsection{Principle of measurement}

In general, the existence of a plasmon is detected as a minimum of reflectance or transmittance. Usually, when the so-called Kretchmann configuration is used (based on attenuated total reflection, ATR), the interrogation is angular, and the measuring parameter is the angle of incidence for which reflectance is minimum. When 
dealing with fibres, spectral interrogation is the most common and the plasmon is associated with a minimum in spectral transmittance (or reflectance in some cases), although some fibre-optic SPR sensors can use intensity as the measuring parameter. Also, spectral interrogations have also been proposed for ATR-based configurations [12-14].

As it can be seen, in the vast majority of SPR sensors reported in the literature, most of the information associated with the plasmon resonance is simply not used, since it is the displacement of a single point, namely that corresponding to a minimum, due to the change in refractive index of the surrounding medium, which is tracked. The shape of the curves is not considered and no other values of spectral or angle transmittance or reflectance are evaluated.

Although plasmon resonances are associated with well-defined dips, it is also true that the width of these dips is not negligible, so we could conceive alternative methods of using the richness of information provided by SPR sensors. For instance, if we consider the value of the spectral transmittance in a SPR fibre sensor for wavelengths different to that of the minimum, it is obvious that these values will vary when the SPR curve is displaced due to the variations of the outer refractive index. In a well-known work, Patrick et al. [15] introduced a concept of measurement for LPGbased sensors that employs this kind of information. A different but related approach can be seen in the works of Suzuki et al. [16,17], where two different, narrow-band, sources are used to provide the pair of interrogating wavelengths for SPR sensors. Two beams are also used in a Kretchmann configuration by Zeng et al. [18].

All these proposals share a common feature: what is used as the measuring parameter in the sensor is no longer the single, welldefined (or not) value of the minimum of the curve, but at least a pair of values placed on each side of that minimum. The increase in the number of parameters is obviously advantageous, since it provides additional degrees of freedom that can be used, for instance, to improve the measurements in terms of independence with respect to spurious influence of other parameters.

However, this concept, powerful as it is, has not been applied up to date, to our knowledge, to the very field where its possibilities are most needed: that of SPR biosensors. These systems are obliged to work in very exigent conditions: since the variations of refractive index induced by the presence of the analytes are always very low, these sensors are very sensitive to any noises or interferences, and must show very good resolution and high sensitivity to be operative. The emphasis in this field has been commonly put in the selection of recognizing agents or in the procedures to immobilize the analyte in the proximity of the transducer, but very little has been done in terms of advanced and efficient interrogation techniques.

To contribute in this sense to the improvement of the performance of plasmon-based sensors, we propose to select two interrogation wavelengths, each on one side of the plasmon dip. Having the complete transmittance spectrum, measured by a CCD spectrometer, we can simply use selected spectral values and combine the detected values in a new measuring parameter. To implement the concept without the need of a spectrometer, we can use the FBGs to measure the power associated with each of these wavelength ranges, which would correspond to the characteristic wavelengths of the gratings. This approach shall permit to process in a most efficient way the information provided by SPR sensors, providing optical power referentiation capability and enabling an increase of the resolution and stability of the measurements. Additionally, by proper localization of the grating pair nearby the sensing head, the reading of the Bragg wavelength of one of the gratings can give the temperature of the sensing volume, which is the critical cross-sensitivity parameter in SPR-based measurements. This interrogation technique of plasmon-based sensors is compatible with an all-fibre deployment of the sensing system, enabling a readily implementation at a reduced cost when compared with those associated with conventional SPR-sensing layouts.

In that sense, we follow the approach introduced by Patrick et al. in the context of interrogation of LPG-based sensors and adapt it to the readout of SPR-sensing structures. For instance, Patrick et al. placed their interrogation wavelengths in the points corresponding to a half-height in an assumed perfectly symmetric spectral dip. In SPR sensors the plasmon resonance curves are not in general symmetric, so an important point of the method is the selection of the interrogation wavelengths looking for the situation where the measurement will show the highest linearity and/or sensitivity. Therefore, these interrogation wavelengths are not necessarily placed symmetrically with respect to the spectral SPR minima, and should be adapted to any particular sensor. Of course, LPG- and SPRbased sensors are different enough to introduce modifications of this kind and, what is more important, to implement an FBG-based interrogation scheme in operative SPR sensors is still a pending task in the highly demanding field of biosensors.

To demonstrate and characterize the proposed interrogation scheme, doubly deposited uniform-waist tapered optical fibres transducers are used. These are efficient SPR structures developed by the authors in the last years [19-21], with very low dependence on light polarization (or no dependence at all for symmetric deposits), and well suited to the demanding requirements of biochemical sensing.

\subsection{Sensor fabrication and experimental set-up}

To fabricate the transducers we use the simple scheme depicted in previous works [19]. We asymmetrically deposited by sputtering in a vacuum chamber an aluminium layer $8 \mathrm{~nm}$ thick and a titanium dioxide layer with different thicknesses between 45 and $50 \mathrm{~nm}$ on a tapered fibre, so that the surface plasmon resonances appear at around $800 \mathrm{~nm}$. Each sensor was fixed to an individual glass support with cyanoacrylate and araldite epoxy, to make the fixation waterproof. The sensors were tested previously [19] in order to determine the behaviour of each SPR sensor when the refractive index of the external medium varies. In this manner, it is possible to select the most suitable ones with respect to the resonance wavelength range of interest. The FBGs fabricated for the interrogation of these SPR sensors present Bragg wavelengths at $809.986 \mathrm{~nm}$ and at $834.62 \mathrm{~nm}$, with a spectral width of $0.6 \mathrm{~nm}$ at half-height. Further FBG wavelengths were limited by the choice of phase masks available, nevertheless, by using the spectra acquired by the $C C D$, acquisition using different narrow spectral intervals could be evaluated. In spite of all, the FBG wavelengths used were appropriated for the SPR sensors available.

The sensors were characterized by measuring their spectral transmittance with a CCD spectrometer, and also by measuring the response of the FBGs with an optical spectral analyser, as it is shown in Fig. 1. The source was a superluminiscent diode (SLD) emitting partially polarized light around $800 \mathrm{~nm}$ with a FWHM around $100 \mathrm{~nm}$. The light is directed to the sensing head and is guided to a directional coupler located in the detection and processing region. One of the outputs of the coupler was connected to the FBGs, and the other one to an Avantes spectrometer (AvaSpec 2048-2) to register the SPR variation. The reflected optical power at the wavelengths selected by the FBGs was directed to an optical spectrum analyser (Ando AQ 6315B).

Although the SPR transducers are in principle polarization dependent, the authors have shown that with tapered structures it is possible to carry out measurements without polarizationcontrolling elements because the SPR minima is deep enough to detect the variations with the same sensitivity [22]. 


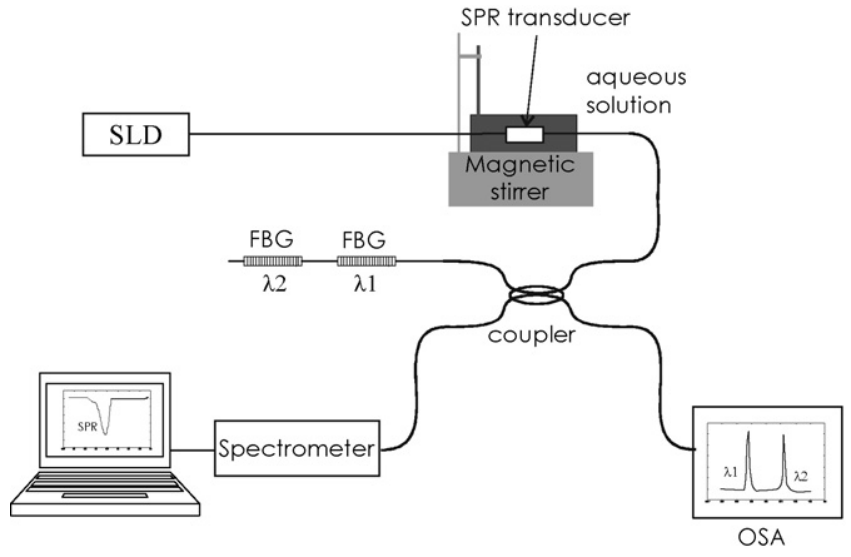

Fig. 1. Scheme of the experimental set-up used for the characterization of the sensors in the laboratory.

The depicted configuration is well suited for a validation of the method and for a use in the laboratory. However, it shall be emphasized that the use of the FBGs permits us to conceive a much simpler operating scheme, in which no spectral analyser and even no spectrometer are needed, and this is one of the most relevant advantages that the method can provide. The proposed scheme for the development of a very compact, portable, and conceptually and operatively very simple device is shown in Fig. 2. The light reflected by the FBGs can be directed to two photodetectors that give signals that with proper processing allows us to obtain the measurand information. Moving a step further, the return fibre could coincide with the illumination fibre just by operating in reflection, which can easily be done by mirroring the fibre end just after the sensing head region. In such case a circulator (or a coupler) would be located nearby the optical source to redirect the return optical power to the FBGs (this configuration is simpler and has the extra advantage of light crossing two times the SPR sensor, therefore enhancing its sensitivity). In this reflection configuration, it is also feasible to consider the location of the FBGs just after the sensing head. In this case, the measurement of the Bragg wavelength shifts of the gratings with standard FBG interrogation equipment will allow to obtain the temperature of the sensing region, a critical cross-sensitivity parameter in SPR-based sensing as mentioned before (with previous calibration, the determination of these shifts allow to compensate for the associated changes in the optical power arriving from the gratings that are due to the temperature induced displacement of the FBGs spectral signatures relative to the plasmon resonance).

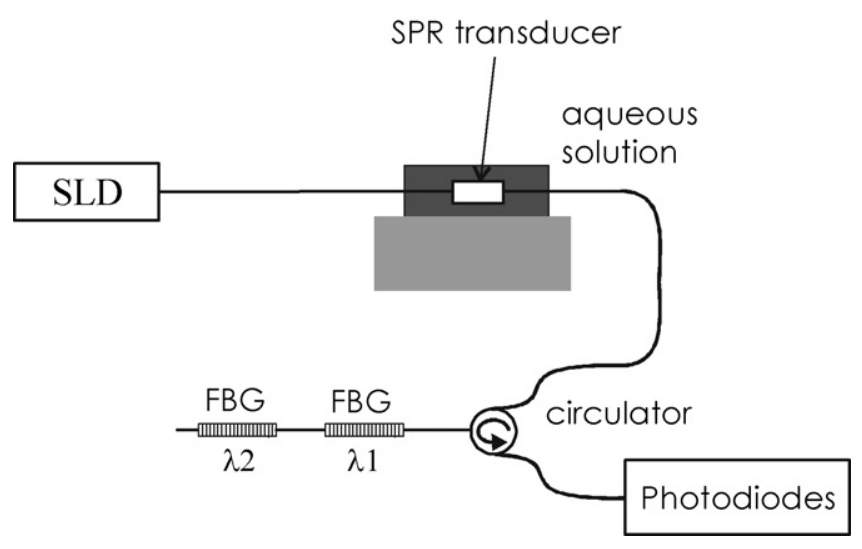

Fig. 2. Scheme of a compact version of the sensor apt for field measurements.

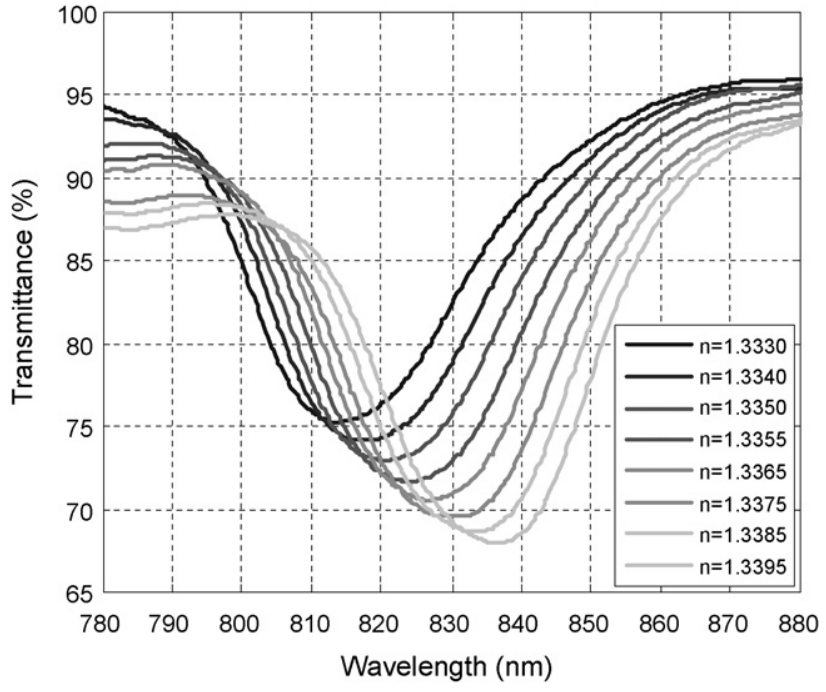

Fig. 3. Variation of the wavelength of the plasmon resonance when the refractive index of the external medium changes.

\section{Experimental results and discussion}

With the above-depicted set-up, the transmittance of SPR and the reflected power of both FBGs were registered at the same time. To carry out the tests, the refractive index of the external medium was changed from 1.333 up to 1.35 , by addition of ethyleneglycol to water. The refractive index value was measured by an Abbe refractometer after each variation in the solution.

With respect to the SPR recorded values, the spectral behaviour of this kind of sensors when SPR appears is shown in Fig. 3. The figure represents the transmittance as a function of the wavelength for several values of the refractive index of the external medium, from 1.333 to 1.34. In Fig. 4 we show the displacement of the minima of these curves as the outer refractive index varies. These are typical experimental curves, and we show some of them, corresponding to different values of refractive index in that range. Some a priori knowledge of the SPR curves is desirable to properly select the Bragg interrogation wavelengths. In principle, it is better to have two wavelengths on the opposite sides of the minimum, although the method can work with any wavelengths. When the minimum

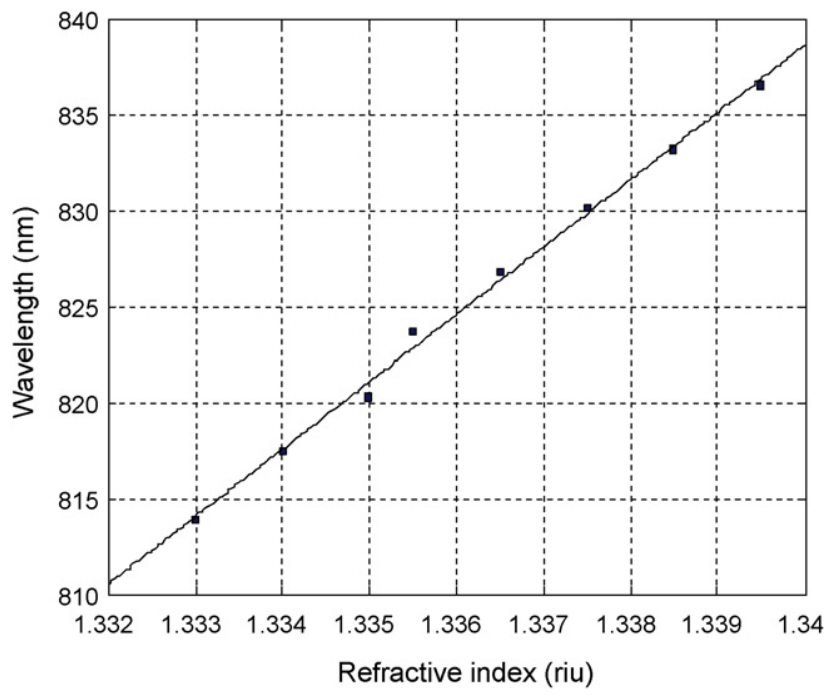

Fig. 4. Variation of the position of the minimum of spectral transmittance of the plasmon curves as a function of the external medium refractive index. 
is displaced, it is still possible to include new interrogation wavelengths to extend the dynamical range of the sensor as much as we want. In our case, as mentioned before, the plasmon resonance wavelengths were located between the Bragg wavelengths of the two FBGs ( $809.986 \mathrm{~nm}$ and $834.62 \mathrm{~nm}$ ) for aqueous solutions.

The information of the two FBGs is used to calculate the normalized power output, given by:

$P_{\text {norm }}=\frac{P_{1}-P_{2}}{P_{1}+P_{2}}$

where $P_{1}$ and $P_{2}$ are the reflected power values for the FBGs 1 and 2 , respectively. In Fig. 5 the variation of this normalized power as a function of the refractive index of the external medium is shown. Since we are working with a subtraction and no modulus is taken, we have some negative values, due to the fact that for some values of refractive index $P_{1}$ can be smaller than $P_{2}$, because the interrogation wavelengths are in opposite sides of the minimum. We represent the values of $P$ for a significant set of refractive indices, in the same range considered above, following the characterization process depicted in the past paragraph. We can see that in this case the curve is not linear, but this is not a significant drawback because its behaviour is stable and can be represented by a polynomial function. Therefore, this calibration data permits to determine the actual refractive index value.

In order to verify the effect of the source power in the measurements, the light in the fibre was varied in an amount of about $8 \%$ by reducing the efficiency of the light injection by means of a piece of glass placed between the source and the fibre. The experiments for higher and lower source power were carried out in an immediate sequence so the changes in the refractive index were exactly the same for both tests. The results are also shown in Fig. 5, in which the normalized power is represented versus the external refractive index for the two optical powers in the system.

It can be seen that the curves are essentially coincident, being the difference around $0.05 \%$. This fact demonstrates the independence of the normalized power with respect to optical power fluctuations, which is an important operational feature for practical application of the SPR-sensing concept.

It is worth mentioning that the shape of these curves is depending on the Bragg wavelengths of the gratings used. In this case it is obtained a nonlinear behaviour except in a small range of the refrac-

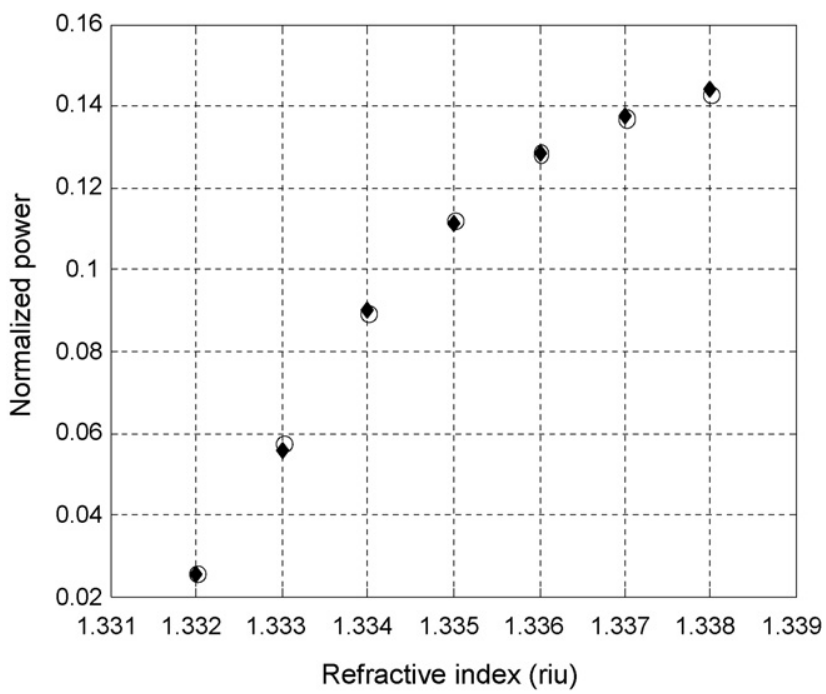

Fig. 5. Variation of the normalized signal as a function of the refractive index of the external medium for two different emitted powers of the optical source. The circles correspond to the higher value of the source power and the solid diamonds to the lower value.

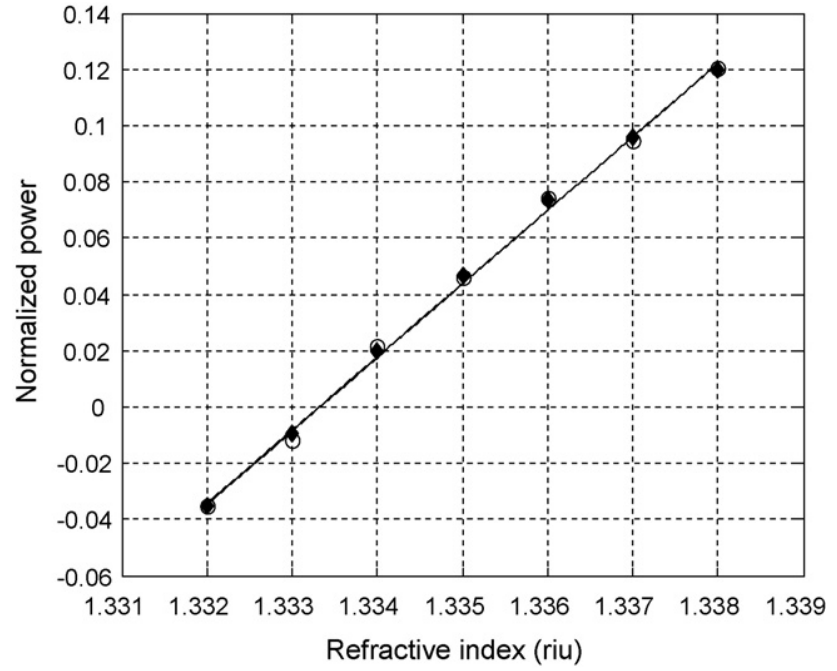

Fig. 6. Variation of the normalized signal as a function of the refractive index of the external medium for two different emitted powers of the optical source using a second set of interrogation wavelengths. The circles correspond to the higher value of the source power (slope: 26.151) and the solid diamonds to the lower value (slope: 26.044).

tive index but another set of FBGs can be chosen so that their Bragg wavelengths are more centred in the linear parts of the SPR minimum, and the behaviour will be linear in a wider range of refractive index. For example, if the first interrogation at $809.986 \mathrm{~nm}$ is maintained but the second one is shifted to $850.08 \mathrm{~nm}$, the variation in this case will be as given in Fig. 6 . Again in this case the two curves are coincident and the difference between their slopes is less than $0.05 \%$. It is possible to conceive a selection technique to choose the ideal interrogation wavelengths in terms of linearity or sensitivity for each different plasmon sensor.

Finally, to know the measurand resolution achievable with this configuration, 10 measurements were registered for each refractive index value for only one of the FBGs (to calculate the dispersion on the value of the maxima without a variation on the refractive index) and for several values of the refractive index (see Fig. 7). The value of the power is decreasing because we have selected the Bragg wavelength on the left of the minimum. The resolution is

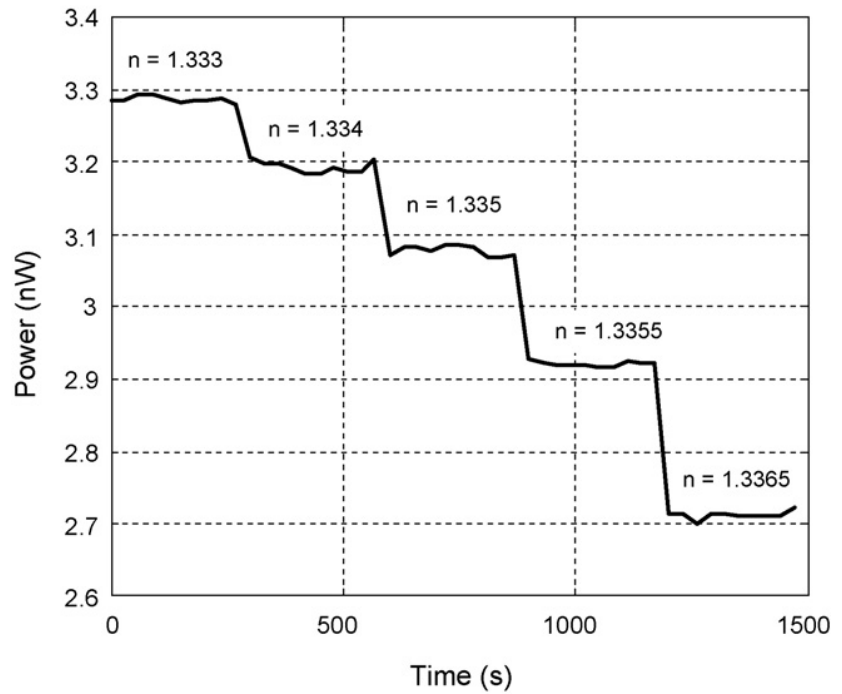

Fig. 7. Variation of the FBG power as a function of time for five different values of the refractive index. 
calculated as:

$\frac{\delta n}{\left(\Delta P_{\text {res }} / \delta P_{\text {noise }}\right)}$

where $\delta n$ is the refractive index variation, $\Delta P_{\text {res }}$ is the power variation that corresponds to that index variation and $\delta P_{\text {noise }}$ is the standard deviation of the power for the 10 measurements at each refractive index value. The value of the resolution varies slightly from one to the other step due to the non-uniformity in the shape of the transmittance curve. The resolution values for all the steps vary from $2 \times 10^{-5}$ to $9 \times 10^{-5}$.

The study of these resolution values reveals the importance of an optimum tuning of the Bragg wavelength of the FBG used to interrogate the sensor. It is better to fit the Bragg wavelength to the region of the curve in which the slope is more abrupt because the corresponding resolution can be as good as $2 \times 10^{-5}$. This means that the sensor interrogation based on fibre Bragg gratings allows to achieve a refractive index resolution that is one order of magnitude better than that obtained with the typical scheme of analysis for these SPR transducers.

Further features of the proposed configuration that will be explored in future works include the multiplexing ability and simultaneous measurement of refractive index and temperature. Multiplexing becomes possible by combining different pairs of FBG wavelengths with each SPR sensor, and simultaneous measurement of temperature and refractive index can be achieved by analysing simultaneously the amplitude and wavelength of the FBGs.

\section{Conclusions}

We have presented a sensing system based on a configuration that incorporates a SPR transducer (doubly deposited uniformwaist tapered fibre) with a dual-FBG interrogation element. This system can be used as a refractometer for the range of refractive indices of aqueous solutions and it is therefore very well suited for the development of biosensors. As the experimental results show, the performance of the system is encouraging, since the resolution of the usual SPR transducers was improved by an order of magnitude and the response of the system was made independent of optical power fluctuations. Additionally, the use of FBGs as interrogating elements also introduces some interesting features and possibilities, since it can provide temperature referencing and multiparameter sensing. Finally, it shall be emphasized that this interrogation scheme can be applied to any SPR transducer.

\section{Acknowledgments}

This work has been partially supported by Spanish Government research project NESTOR, ref. CTM2004-03899; Comunidad de Madrid research project FUTURSEN, ref. S-0505/AMB-0374 and by Proyecto de Investigación Santander/Complutense, ref. PR34/0715886. This work was supported partially by the Portuguese Government - Fundação para a Ciência e Tecnologia (FCT) through the grant SFRH/BD/30086/2006. N. Díaz-Herrera is thankful for the grant within the program 'Becas Internacionales Universidad Complutense/Empresa Flores Valles 2008'.

\section{References}

[1] J. Homola, S.S. Yee, G. Gauglitz, Surface plasmon resonance sensors: review, Sensors and Actuators B 54 (1999) 3-15.

[2] A.K. Sharma, R. Jha, B.D. Gupta, Fiber-optic sensors based on surface plasmon resonance: a comprehensive review, IEEE Sensors Journal 7 (8) (2007) 1118-1129.

[3] A. Leung, P.M. Shankar, R. Mutharasan, A review of fiber-optic biosensors, Sensors and Actuators B 125 (2007) 688-703.
[4] R. Kashyap, Fiber Bragg Gratings, Academic Press, San Diego, 1999.

[5] A. Othonos, Fiber Bragg gratings, Review of Scientific Instruments 68 (12) (1997) 4309-4341.

[6] B. Lee, Review of the present status of optical fiber sensors, Optical Fiber Technology 9 (2003) 57-79.

[7] J.M. López-Higuera, Handbook of Optical Fibre Sensing Technology, John Wiley \& Sons, New York, 2002

[8] G. Nemova, R. Kashyap, Fiber-Bragg-grating-assisted surface plasmonpolariton sensor, Optics Letters 31 (14)(2006) 2118-2120.

[9] J. Ctyroky, W. Ecke, K. Schroeder, R. Slavik, Separation of refractive index and temperature measurements using surface plasmon-coupled fiber grating, Proceedings of the SPIE 4185 (2000) 322-325.

[10] T. Allsop, R. Neal, S. Rehman, D.J. Webb, D. Mapps, I. Bennion, Generation of infrared surface plasmon resonances with high refractive index sensitivity utilizing tilted fiber Bragg gratings, Applied Optics 46 (22) (2007) 5456-5460.

[11] F. Abdelmalek, Surface plasmon resonance based on Bragg gratings to test the durability of Au-Al films, Materials Letters 57 (2001) 213-218.

[12] A.C.J. Tubb, F.P. Payne, R.B. Millington, C.R. Lowe, Single-mode optical fibre surface plasma wave chemical sensor, Sensors and Actuators B 41 (1997) 71-79.

[13] F.J. Bueno, Ó. Esteban, N. Díaz-Herrera, M.C. Navarrete, A. González-Cano, Sensing properties of asymmetric double-layer covered tapered fibres, Applied Optics 73 (2001) 95-99.

[14] J. Homola, Present and future of surface plasmon resonance biosensors, Anlytical and Bioanalytical Chemistry 377 (2003) 528-539.

[15] H.J. Patrick, G.M. Williams, A.D. Kersey, J.R. Pedrazzani, A.M. Vengsarkar, Hybrid fiber Bragg grating/long period fiber grating sensor for strain/temperature discrimination, IEEE Photonics Technology Letters 8 (9) (1996) 1223-1225.

[16] A. Suzuki, J. Kondoh, Y. Matsui, S. Shiokawa, K. Suzuki, Development of novel optical waveguide surface plasmon resonance (SPR) sensor with dual light emmiting diodes, Sensors and Actuators B 106 (2005) 383-387.

[17] H. Suzuki, M. Sugimoto, Y. Matsui, J. Kondoh, Fundamental characteristic of a dual-colour fibre optic SPR sensor, Measurement Science and Technology 17 (2006) 1547-1552.

[18] H. Zeng, X. Wang, X. Zhang, Studies of surface plasmon resonance sensor using bi-beam differential measurement approach, Journal of Zheijang University Science A 8 (12) (2007) 2027-2031.

[19] A. González-Cano, F.J. Bueno, Ó. Esteban, N. Díaz-Herrera, M.C. Navarrete, Multiple surface-plasmon resonance in uniform-waist tapered optical fibers with an asymmetric double-layer deposition. Applied Optics 44 (4) (2005) 519-526.

[20] Ó. Esteban, N. Díaz-Herrera, M.C. Navarrete, A. González-Cano, SPR sensors based on uniform-waist tapered fibers in reflective configuration, Applied Optics 45 (28) (2006) 7294-7298.

[21] Ó. Esteban, A. González-Cano, N. Díaz-Herrera, M.C. Navarrete, Absorption as a selective mechanism in surface plasmon resonance fiber optic sensors, Optics Letters 31 (21) (2006) 3089-3091.

[22] A González-Cano, N. Díaz-Herrera M.C. Navarrete Ó. Esteban, DL-UWTs: novel devices for chemical and biological sensing, Proceedings of the SPIE 6619 (2007), 66191S-1/4

\section{Biographies}

Natalia Díaz-Herrera received the MSc degree in Physics from the Universidad Autónoma de Madrid, Madrid, Spain, in 2001 and the PhD degree in applied optics from the Universidad Complutense de Madrid (UCM) in 2005. In 2006 she joined the Optics Department of the UCM as a lecturer, where she has carried out her research and teaching activities. She has been involved in several European and Spanish projects. Her research interests are optical fibre sensors, specifically for environmental applications.

Diana Viegas graduated in physics in 2003 and received the MSc degree in computational methods in science and engineering in 2006, both from the University of Porto, Porto, Portugal. Her main interests are optical physics, including interferometry. She is doing her PhD work on microfabrication of optical devices for sensing applications in Faculty of Sciences of University of Porto since 2007.

Pedro A.S. Jorge graduated in Applied Physics (Optics and Lasers) at the University of Minho (1996), MSc in Optoelectronics and Lasers at the Physics Department of University of Porto (2000) with dissertation on optical current sensors for high voltage operation; in 2006 he concluded his PhD program at Porto University in collaboration with the Department of Physics and Optical Sciences at the University of Charlotte, North Carolina, USA, with work developed in luminescence based optical fibre systems for biochemical sensing applications; the work involved studies of the applicability of luminescent nanoparticles as tools for biochemical sensing. Pedro Jorge is currently a Senior Researcher at INESC Porto where he leads a small team aiming to explore the potential of optical fibre and integrated optics technologies in the development of biochemical sensors for environmental and medical applications. He published approximately 20 journal and conference papers and holds one patent.

Francisco M. Araújo graduated in 1993 in Physics (Optics and Electronics) from the University of Porto, Portugal, where he received his PhD in Physics in 2000. He is a senior researcher with INESC Porto. His main activity involves fibre-optic sensing and optical communications and. He is also a cofounder of FibreSensing, an INESC Porto spin-off company developing fibre-optic sensors and monitoring systems. 
José-Luis Santos graduated in Physics (Optics and Electronics) by University of Porto (1983). PhD from the same University (1993). His main research interests are related to optical fibre technology and optical fibre sensing. He holds the position of Associate Professor of Physics Department of University of Porto, and he is also manager of INESC Porto Optoelectronics and Electronic Systems Unit. He is member of OSA and SPIE.

María-Cruz Navarrete received her MSc degree in Physics in 1986, and her $\mathrm{PhD}$ degree of Physics in 1994 in the Universidad Complutense de Madrid (UCM, Spain) She joined Optics Department (UCM) in 1990 where she has carried out her research and teaching activities. She has been involved in fibre-optic research and in severa European and Spanish projects. During the last 12 years she has been working in optical fibre sensors, mainly applied to environmental measurements. Nowadays she is an assistant professor in the Optics Department and a member of the Applied Optics Complutense research group.
Agustín González-Cano was born in Madrid (Spain) in 1964. He received his MSc in Physics (1987) and PhD in Advanced Optics (1994) in the Universidad Complutense de Madrid (UCM), where he has developed his research and teaching activities during the last 16 years. Currently he is Professor in the School of Optics and Optometry of the UCM, where his main teaching subjects are Ophthalmic Optics and Geometrical Optics. His research interests are basically optical sensors, and specifically fibre-optic sensors for environmental applications. In the past he has also been working in image processing, fringe pattern analysis and photoelasticity. In recent times he is also interested in research on the history of Optics. He has published more than 30 papers and has been involved in many different research projects, including some European Union-funded ones. He is a member of the Spanish Optics Association (SEDO). 